\section{The diagnostic role of Next Generation Sequencing in uncovering isolated splenomegaly: A case report}

\author{
Giuseppe Auteri, ${ }^{1}$ Daniela Bartoletti, ${ }^{1}$ \\ Clara Bertuzzi, ${ }^{2}$ Francesco Bacci, ${ }^{2}$ \\ Valeria Tonini, ${ }^{3}$ Lucia Catani, ${ }^{1}$ \\ Nicola Vianelli, ${ }^{1}$ Michele Cavo, ${ }^{1}$ \\ Francesca Palandri ${ }^{1}$ \\ ${ }^{1}$ IRCCS Azienda Ospedaliero- \\ Universitaria di Bologna, Istituto di \\ Ematologia "Seràgnoli", Bologna; \\ ${ }^{2}$ Hematopathology Unit, ${ }^{3}$ Emergency \\ Surgery Unit, Sant'Orsola-Malpighi \\ University Hospital, Bologna, Italy
}

\begin{abstract}
Many diseases can induce splenomegaly, however, about $5 \%$ of splenomegalies are idiopathic. When there is no underlying treatable cause, and the splenomegaly significantly affects the quality of life, splenectomy is the best therapeutic choice. A 67-year-old woman had idiopathic and asymptomatic splenomegaly. The increase in splenomegaly resulted in hypersplenism with cytopenia and symptoms related to abdominal discomfort. The patient underwent splenectomy which led to clinical improvement. A histological examination showed the presence of hematopoietic tissue. Peripheral blood Next Generation Sequencing with the myeloid panel SOPHiA Genetics showed the following mutations: ASXL1, SRSF2, KRAS and TET2. Three out of these four mutations were also found in the splenic tissue. Next Generation Sequencing could be useful in the diagnosis of splenomegalies associated with myeloproliferative neoplasms otherwise defined as idiopathic, in order to address a therapeutic strategy.
\end{abstract}

\section{Introduction}

Splenomegaly is defined as spleen weight above $250 \mathrm{~g},{ }^{1}$ however various methods allow an indirect definition of splenomegaly (for example the longitudinal diameter over $11-14 \mathrm{~cm}$, the spleen volume more than $314.5 \mathrm{~cm}^{3}$ calculated by ultrasound or CT). ${ }^{2,3}$ Splenomegaly is often a condition secondary to other diseases (Table 1). ${ }^{4}$ The differential diagnosis is often challenging and requires the involvement of numerous tools in terms of blood chemistry, histology and imaging. 5,6

The spleen has a role in presenting antigens: an infection can stimulate a reactive splenomegaly, often reversible at the disruption of an antigenic stimulus. Consistently, even an autoimmune antigenic stimulus could result in reactive splenomegaly.

The spleen is in charge of the disposal of blood cells. Congenital blood cell disorders can cause splenomegaly, particularly during the first decades of life.

Congestion of portal venous flow or congestive heart failure may be responsible for splenomegaly, resulting in hypersplenism and consequent cytopenia. Hyperaccumulation disorders are congenital or acquired and may induce organ failure or increase in volume. These diseases are usually associated with symptoms concerning other organs.

Neoplasms can induce splenomegaly due to metastasis or primitive splenic neoplasms, especially hematologic ones. ${ }^{7}$ Despite these causes, in about $5 \%$ of cases, splenomegaly remains idiopathic. ${ }^{5}$

In hematology, splenomegaly often underlies a neoplastic disease and its identification leads to a diagnosis and, therefore, to a therapeutic approach.

Potentially all lymphomas can be located in the spleen. Splenomegaly sometimes represents the first or the only sign of neoplasia. Except in rare cases (e.g. in splenic marginal zone lymphoma) splenectomy is not recommended and therapy of the underlying disease solves the splenomegaly.

Chronic Myeloid Leukemia (CML) is symptomatic in only $50 \%$ of cases, but splenomegaly is a frequent sign detected at diagnosis and the spleen size measured from the left costal margin is fundamental to calculate the Sokal score. ${ }^{8}$ The JAK $1 / 2$ inhibitor ruxolitinib was introduced into clinical practice, reducing spleen size and improving the quality of life in most patients. $^{9}$

The diagnosis of MPNs were redefined by the WHO in 2016, giving a central role to the detection of JAK2,CALR and MPL mutations and to the histological examination of the bone marrow. ${ }^{10}$ About 2\% of patient with Polycythemia Vera and $10 \%$ of patient with Essential Thrombocythemia and Myelofibrosis do not carry any of these mutations.

The so called "triple negative" patients show a worse prognosis in Leukemia Free Survival and the comprehension of the pathogenesis, diagnosis and management of these patients still represent an unmet clinical need. ${ }^{11}$

Next Generation Sequencing (NGS) allows the simultaneous analysis of a large
Correspondence: Giuseppe Auteri, Department of Experimental Medicine, IRCCS Azienda Ospedaliero-Universitaria di Bologna, Istituto di Ematologia "Seràgnoli", Bologna, Via Giuseppe Massarenti, 9, Bologna 40138, Italy.

Tel.: +39.051.2143834

E-mail: giuseppe.auteri2@unibo.it

Key words: Splenomegaly, Next Generation Sequencing, Myeloproliferative Neoplasms.

Contributions: The authors contributed equally. Conflict of interest: GA, FB, DB, CB, LC, VT, $\mathrm{NC}$ declare no potential conflict of interest, $\mathrm{MC}$ reports personal fees and other from Janssen, Celgene, Novartis, Bristol-Myers Squibb, Amgen, Takeda, outside the submitted work; FP reports personal fees and other from AOP Orphan Pharmaceuticals, Celgene, Novartis, outside the submitted work.

Funding: None.

Ethics approval: The paper was approved by the local ethics committee.

Consent to publication: Received.

Availability of data and materials: In respect of privacy, it is possible to consult the material and data of the case report

Received for publication: 27 July 2020.

Revision received: 4 November 2020.

Accepted for publication: 1 February 2021.

This work is licensed under a Creative Commons Attribution-NonCommercial 4.0 International License (CC BY-NC 4.0).

${ }^{\circ}$ Copyright: the Author(s), 2021

Licensee PAGEPress, Italy

Hematology Reports 2021; 13:8814

doi:10.4081/hr.2021.8814

quantity of samples and genes. The "Myeloid Solution" panel of SOPHiA genetics (Sophia Genetics, Saint Sulpice, Switzerland) analyzes 30 genes commonly involved in myeloproliferative neoplasms. Therefore, it is possible to have a lot of specific information about a patient in a relatively short time and at lower costs than techniques in which specific single genes are searched.

NGS permits to identify MPN patients with worse prognosis, especially "triple negative" ones and its use as a diagnostic tool is increasing. ${ }^{12}$

\section{Case Report}

We describe the case of a 67-year-old female that came to our observation because of a random detection of 
splenomegaly (Figure 1).

In June 2017, the patient suffered from a severe dyspnea (NYHA class II/III) and asthenia. Complete blood count and laboratory evaluations were normal with the exception of a not clinically significant monoclonal $\mathrm{IgG} / \mathrm{k}$ type peak (295 mg/dl), a rheumatoid factor and anti-nucleic autoanti- bodies. The patient had a multinodular thyroid goiter, she was obese and suffered from Sjogren disease requiring no therapy. The patient underwent a PET/CT which highlighted nodular overfixing to the medium lung lobe, bones and spleen. Bronchial cyto-morphology showed follicular bronchitis with peri-bronchial metaplasia and foci of interstitial lymphoplasmacellular pneumonia. CT scan revealed a spleen longitudinal diameter of $25 \mathrm{~cm}$, triggering a hematologic diagnostic work-up. On examination, grade 2 splenomegaly $(2 \mathrm{~cm}$ from the costal arch) was noted. Thoracic and neurological examination were negative and there were no signs of heart failure.

Table 1. Causes of splenomegaly.

\begin{tabular}{ll} 
Causes of splenomegaly & Specific diseases \\
$\begin{array}{l}\text { Acute infection/infestation } \\
\text { Subacute or chronic infection/infestation }\end{array}$ & Mononucleosis, Viral Hepatitis, Septicemia, Typhus, Toxoplasmosis \\
\hline Autoimmune diseases & Bacterial endocarditis, Brucellosis, Syphilis, HIV/AIDS, Plasmodium, Leishmania, Schistosoma \\
Congenital blood cells disease & $\begin{array}{l}\text { Rheumatoid Arthritis, Systemic Lupus Erythematosus, Rheumatic Polymyalgia, Systemic Sclerosis, } \\
\text { Primitive Biliary Cirrhosis }\end{array}$ \\
\hline Systemic disease & Hereditary Spherocytosis, Thalassemia, Sickled Cells disease \\
Hyperaccumulation disorders & Congestive Heart Failure, Portal Hypertension \\
Cancer & Sarcoidosis, Gaucher's syndrome, Amyloidosis, Niemann-Pick syndrome, Wagner's Granulomatosis \\
Idiopathic & Lymphomas, Chronic Myeloproliferative Neoplasms, Myelofibrosis, Polycythemia Vera, \\
\end{tabular}

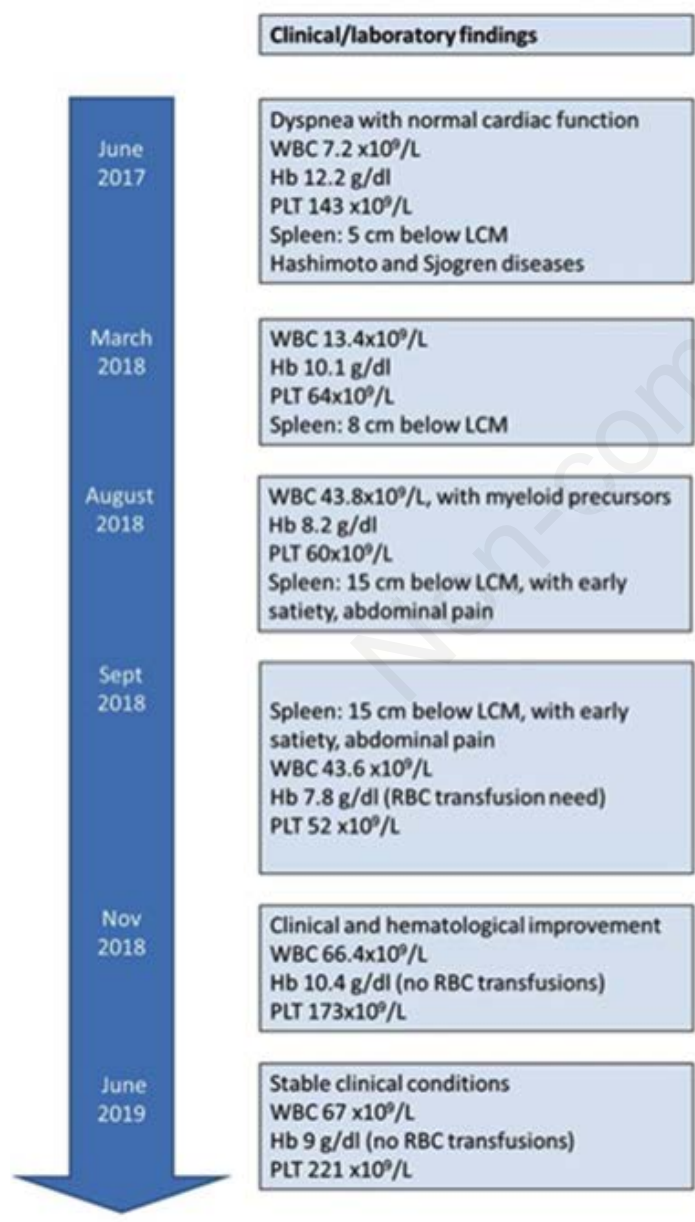

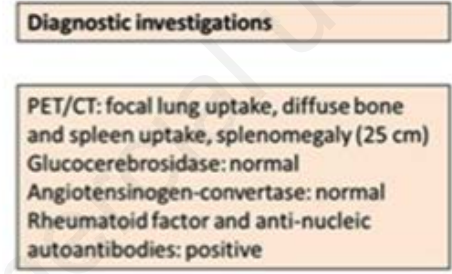

Bone marrow biopsy: reactive pattern Peripheral blood PCR: BCR-ABL absent; $\angle A K Z^{* 11 \%}$, CALR and MPL wild-type
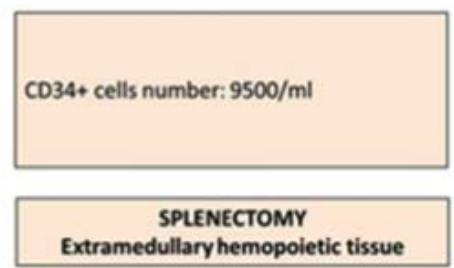

Peripheral blood NGS: mutations of ASXL1, SRSF2, KRAS, TET2 Spleen NGS: mutations of SRSF2, KRAS, TET2

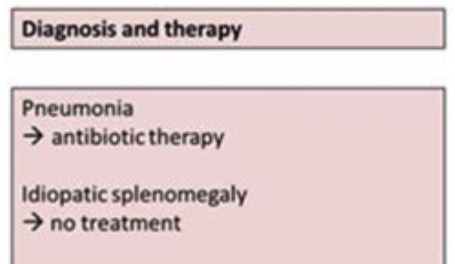

Idiopatic splenomegaly, asymptomatic $\rightarrow$ no treatment

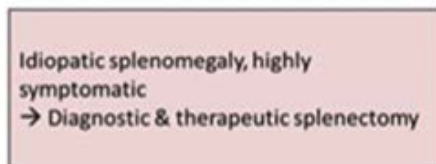

Possible diagnosis of chronic myeloproliferative neoplasms . unclassified

Diagnosis of clonal myeloid neoplasm
Hydroxyurea therapy (high thrombotic risk
\& hyperleukocytosis)

Hydroxyurea therapy

Figure 1. Case report timeline Clinical/laboratory findings (left), diagnostic investigations (center), diagnostic/therapeutic approach (right), and timing (arrow). WBCs: White blood cells; Hb: hemoglobin; PLT: platelets; LCM: left costal margin; RBCs: red blood cells; PCR: polymerase chain reaction. 
Infective and active autoimmune diseases were excluded.

Abdominal ultrasound showed an increased size and stiffness of the hepatic parenchyma $(67 \mathrm{kPa})$ with finely irregular profiles, thickened and granular echo-structure, arteriovenous hepatic fistula, hepatic lymph nodes with a reactive appearance, a splenic longitudinal diameter of $26 \mathrm{~cm}$ and signs of portal hypertension.

The $B C R-A B L 1$ rearrangement and the mutations of JAK2V617F, CALR and MPL were absent.

In March 2018, mild leukocytosis $\left(13.4 \times 10^{9} / \mathrm{L}\right)$ with normal distribution of leucocytes subtype, anemia $(10.1 \mathrm{~g} / \mathrm{dl})$ and thrombocytopenia $\left(64 \times 10^{9} / \mathrm{L}\right)$ occurred. No leukoerythroblastosis was detected and red blood cells shapes were normal.

A bone marrow biopsy was performed. The tissue was highly hyper-cellulated (98\%), with a marked expansion of the granulopoiesis, still well maturating, erythropoiesis was reduced, megakaryocytes did not have bulbous nuclei and were not organized in clusters as in Myelofibrosis. Fibrosis was absent (grade 0). These histological features did not fulfil criteria for an MPN diagnosis nor a Myelodysplastic syndrome but were consistent with an inflammatory/reactive pattern related to cirrhosis. No evidence of lymphoid nor solid neoplasm was found. Cell morphology and cytogenetics could not be performed due to dry tap.

The patient's general condition underwent rapid changes during the following months. In August 2018 she became transfusion-dependent $(\mathrm{Hb}<8 \mathrm{~g} / \mathrm{dl})$, leukocytes reached $43.8 \times 10^{9} / \mathrm{L}$, of which $41 \%$ were myeloid precursors, with no blast cell. The slight excess of CD34 + leukocytes in peripheral blood, detected with flow cytometry, was not suggestive for a diagnosis of Myelofibrosis with an MPN $(0.11 \%$ of total peripheral cells).

Overall, the clinical and hematological picture was deemed to be secondary to cryptogenic cirrhosis. Splenomegaly further increased (from g III to g IV) and became symptomatic. Since the patient was transfusion-dependent and suffered from early satiety, splenic bulkiness, dyspnea and other symptoms related to anemia, she underwent splenectomy firstly with therapeutic purpose.

Splenectomy resulted in an improvement of anemia $(10.4 \mathrm{~g} / \mathrm{dl})$, thrombocytopenia $\left(173 \times 10^{9} / \mathrm{L}\right)$ and symptoms.

The histological examination of the spleen, that had reached the maximum size of $35 \mathrm{~cm}$, showed an extensive architectural effacement given by extramedullary hemopoietic tissue which replaced most of the regular splenic parenchyma (Figure 2).

At the time of splenectomy, a blood sample was collected for the search of myeloid mutations according to the "Myeloid Solution" panel of SOPHiA genetics. We observed a duplication in exon 12 of the ASXL1 gene with $30 \%$ of allele burden (p.Gly64Thr frameshift, c.1934dupG), a missense mutation in exon 1 of the $S R S F 2$ gene with $50 \%$ of allele burden (p.Pro95Arg, c.284C $>\mathrm{G}$ ), a missense mutation in exon 2 of the KRAS gene with $46 \%$ of allele burden (p.Gly12Arg, c. $34 \mathrm{G}>\mathrm{C}$ ) and a missense mutation in exon 6 of the TET2 gene with $54 \%$ of allele burden (p.Cys1263Tyr, c.3788G $>$ A).

Since hematopoietic tissue had been found in the spleen and the patient showed neutrophilic leukocytosis, as often happens in the MPN, we searched for the same mutations in the splenic tissue

DNA was extracted from the formalinfixed and paraffin-embedded samples and tested by control gene PCR (100-400 bp amplicons) in order to verify its integrity. Splenic DNA was analyzed with NGS. Three out of four of the mutations reported in peripheral blood were found (SRSF2, KRAS and TET2). The ASXL1 mutation probably was not found due to difficulties related to the DNA extraction method and to lower allelic burden.

The current patient's clinical conditions are good. Splenomegaly-related symptoms are no longer present. The patient is anemic but no longer in need of transfusion. Hydroxyhurea is ongoing to control leukocytosis. The platelet count has normalized.

\section{Discussion}

Ruxolitinib is effective in reducing splenomegaly in most patients with MF and PV. ${ }^{9,13}$ However, in MF patients who are refractory to medical therapies, splenectomy may be useful to temporarily improve cytopenia and symptoms. ${ }^{14}$ In the present clinical case, the use of ruxolitinib was not possible due to the lack of a diagnosis of MPN and thrombocytopenia. A spleen biopsy would have exposed the patient to a high risk of bleeding. Because of the worsening of cytopenia and splenomegaly-related symptoms, splenectomy became the only executable treatment as well as diagnostic tool. ${ }^{15}$

In this case, NGS was crucial for diagnostic definition of a splenomegaly associated with inconclusive marrow histology and molecular findings.
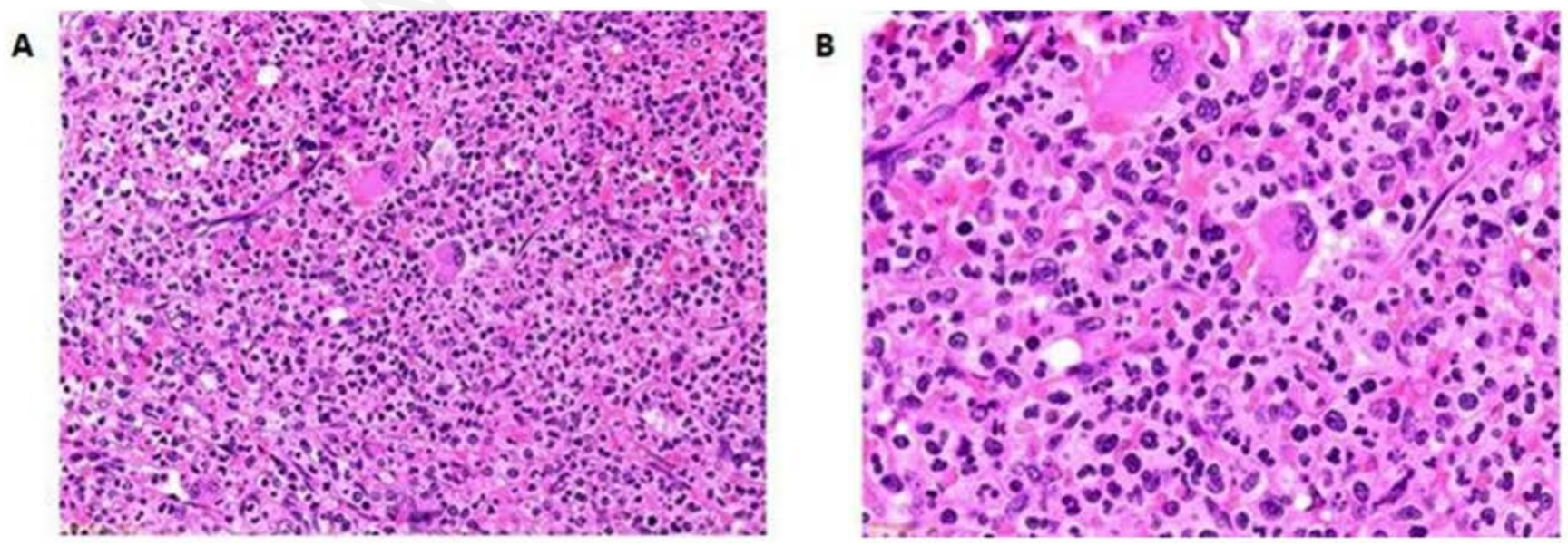

Figure 2. Splenic architectural effacement given by the heterotopic hemopoietic tissue with evident megakaryocytes (Hematoxylin and eosin staining). A) $20 \mathrm{X}$ magnification; B) $40 \mathrm{X}$ magnification. 


\section{Conclusions}

NGS may represent a useful diagnostic and prognostic tool for patients with unexplained splenomegaly. Its implementation in internal medicine departments may be important to improve the management of patients with idiopathic splenomegaly.

In MPNs, the use of NGS helps to better stratify patients according to risk category and to choose more efficient therapeutic approaches such as hematopoietic stem cell transplantation. ${ }^{16-18}$

In Myelofibrosis, the detection of ASXL1, EZH2, SRSF 2 or IDH1/2 mutations define a high-risk patient. ${ }^{12}$ The ELN suggests transplantation in patients carrying high risk mutations, even when at intermediate-1 IPSS/DIPSS risk. ${ }^{18}$

Evidence of a specific mutation would help identify specific drugs. For instance, a $K R A S$-inhibitor that may be useful in our patient has been developed. ${ }^{19}$ Overall, the systematic research of myeloid panel mutations in NGS may improve the knowledge on MPNs pathogenesis and provide crucial information for clinical and therapeutical decisions.

\section{References}

1. Grover SA, Barkun AN, Sackett DL. The rational clinical examination. Does this patient have splenomegaly? JAMA 1993;270:2218-21.

2. Yetter EM, Acosta KB, Olson MC, Blundell K. Estimating splenic volume: sonographic measurements correlated with helical CT determination. AJR Am J Roentgenol 2003;181:1615-20.

3. Bezerra AS, D'Ippolito G, Faintuch S, et al. Determination of splenomegaly by $\mathrm{CT}$ : is there a place for a single measurement? AJR Am J Roentgenol
2005; 184:1510-3.

4. Pozo AL, Godfrey EM, Bowles KM. Splenomegaly: investigation, diagnosis and management. Blood Rev 2009;23:105-11.

5. Sjoberg BP, Menias CO, Lubner MG, et al. Splenomegaly: A Combined Clinical and Radiologic Approach to the Differential Diagnosis. Gastroenterol Clin North Am 2018;47:643-66.

6. Motyckova G, Steensma DP. Why does my patient have lymphadenopathy or splenomegaly? Hematol Oncol Clin North Am 2012;26:395-408.

7. Bonnet S, Guédon A, Ribeil JA, et al. Indications and outcome of splenectomy in hematologic disease. J Visc Surg 2017;154:421-9.

8. Sokal JE, Cox EB, Baccarani M, et al. Prognostic discrimination in «goodrisk» chronic granulocytic leukemia. Blood 1984;63:789-99.

9. Mesa RA, Gotlib J, Gupta V, et al. Effect of ruxolitinib therapy on myelofibrosis-related symptoms and other patient-reported outcomes in COMFORT-I: a randomized, doubleblind, placebo-controlled trial. J Clin Oncol Off J Am Soc Clin Oncol 2013;31:1285-92.

10. Arber DA, Orazi A, Hasserjian R, et al. The 2016 revision to the World Health Organization classification of myeloid neoplasms and acute leukemia. Blood 2016;127:2391-405.

11. Tefferi A, Guglielmelli P, Larson DR, et al. Long-term survival and blast transformation in molecularly annotated essential thrombocythemia, polycythemia vera, and myelofibrosis. Blood 2014;124:2507-13.

12. Guglielmelli P, Lasho TL, Rotunno G, et al. MIPSS70: Mutation-Enhanced International Prognostic Score System for Transplantation-Age Patients With
Primary Myelofibrosis. J Clin Oncol Off J Am Soc Clin Oncol 2018;36:310 8.

13. Passamonti F, Griesshammer M, Palandri F, et al. Ruxolitinib for the treatment of inadequately controlled polycythaemia vera without splenomegaly (RESPONSE-2): a randomised, open-label, phase $3 \mathrm{~b}$ study. Lancet Oncol 2017;18:88-99.

14. Mesa RA, Tefferi A. Palliative splenectomy in myelofibrosis with myeloid metaplasia. Leuk Lymphoma 2001;42:901-11.

15. Pottakkat B, Kashyap R, Kumar A, et al. Redefining the role of splenectomy in patients with idiopathic splenomegaly. ANZ J Surg 2006;76:679-82.

16. Bacher U, Shumilov E, Flach J, et al Challenges in the introduction of nextgeneration sequencing (NGS) for diagnostics of myeloid malignancies into clinical routine use. Blood Cancer J 2018;8:113.

17. Alduaij W, McNamara CJ, Schuh A, et al. Clinical Utility of Next-generation Sequencing in the Management of Myeloproliferative Neoplasms: A Single-Center Experience. HemaSphere 2018;2:e44.

18. Barbui T, Tefferi A, Vannucchi AM, et al. Philadelphia chromosome-negative classical myeloproliferative neoplasms: revised management recommendations from European LeukemiaNet. Leukemia 2018;32:1057-69.

19. Ostrem JML, Shokat KM. Direct smallmolecule inhibitors of KRAS: from structural insights to mechanism-based design. Nat Rev Drug Discov 2016;15:771-85. 